\title{
Adoption d'une culture contraceptive et maîtrise du projet familial. La contraception constitue-t-elle une Capabilité ?
}

\section{Claudine Sauvain-Dugerdil', Nedialka Douptcheva² and Sory Ibrahima Diawara ${ }^{3}$}

${ }^{1}$ Institut d'études démographiques et du parcours de vie (I-DEMO), Université de Genève, Claudine.Sauvain@unige.ch

${ }^{2}$ Harvard School of Public Health (Cambridge, USA) and I-DEMO (University of Geneva)

${ }^{3}$ Dept de santé publique, Faculté Médecine, Université de Bamako

\section{Résumé}

Nous examinons les facteurs d'adoption d'une "culture contraceptive ", décrite par la connaissance, la pratique et les intentions, et ses liens avec l'espacement des naissances. L'analyse des enquêtes EDS Mali (2006) et Ghana (2008) montre la persistance du fossé entre connaissance et pratique - la connaissance de la contraception ne gomme pas les inégalités de pratique - et le rôle des "facteurs de conversion". Ainsi l'avantage des habitants des grandes villes disparaît lorsque l'on contrôle pour les caractéristiques personnelles. Les comportements des femmes apparaissent influencés par la réalité de leur situation familiale, alors que ceux des hommes reposeraient plus sur des valeurs. Finalement, ce n'est pas l'adoption de la contraception qui importe pour l'espacement des naissances, mais le profil des femmes qui l'adoptent : la contraception ne constitue pas une Capabilité - une dimension de la liberté de planifier sa famille - mais un moyen parmi d'autres pour espacer les naissances.

Mots-clés: culture contraceptive, espacement des naissances, Mali, Ghana, EDS.

\section{Abstract}

\section{Adoption of a contraceptive culture and control of the family plans. Can contraception be considered as a Capability?}

We examine family planning in terms of the adoption of a "contraceptive culture", as described by knowledge, practice and intentions, and its links with birth spacing. Analyses of DHS from Mali (2006) and Ghana (2008) confirm the persistence of a large gap between knowledge and practice - knowledge does not erase inequalities in practice - and the role of "conversion factors". In particular, the advantage of the residents of large cities disappears when controlling for personal characteristics. Gender differences are consistent, especially in Mali, with a higher dependency of women on their actual situation, while men's behaviour seems to rely more on their values. Last, contraceptive culture appears only weakly associated with birth spacing: more than adopting contraception, what counts are the characteristics of those women who adopt it. Contraception is not a Capability - a dimension of the freedom to plan the family - but one means among others for birth spacing.

Keywords: contraceptive culture, birth spacing, Mali, Ghana, DHS. 


\section{Introduction: Ré-examiner le rôle de}

\section{la contraception}

Le débat entre partisans des politiques de contrôle des naissances et des politiques de population au sens large qui, dans la perspective du paradigme de la CIPD, donnent priorité à la situation des femmes et à leur qualité de vie, a été récemment rouvert. Parallèlement à la réaffirmation des objectifs de la Conférence internationale sur la Population et le Développement (CIPD, 1994) dans le cadre du bilan des 20 ans de son programme d'action, émerge un nouvel activisme pour le planning familial, avec une série de conférences, dont le sommet de Londres en juillet 2012 qui a lancé l'initiative FP 2020'. Cet activisme est l'expression des conséquences paradoxales de la prise en compte de la santé de la reproduction dans les Objectifs du Millénaire pour le Développement (OMD) et la fixation d'objectifs quantitatifs en termes de prévalence contraceptive et de besoins non satisfaits en contraception. La SR est ainsi reconnue comme une dimension centrale de la qualité de vie, mais elle s'inscrit dans ce que Yamin et Boulanger (2013) décrivent comme le modèle de développement promu par une mise en œuvre à travers des objectifs quantitatifs qui, conçus comme indicateurs de suivi, se sont mués en objectifs politiques. Les défenseurs de la santé de la reproduction dénoncent le risque "d'une porte ouverte vers le retour à la mentalité discréditée de 'contrôle de la population' et de mesures coercitives sur le terrain" (Cohen, 2012).

On assiste donc à la réémergence d'une vision qui met l'offre de contraception au centre, comme la clé pour à la fois diminuer la fécondité et promouvoir la situation de la femme. Les besoins non satisfaits sont devenus un " concept organisationnel des politiques de population " (Casterline and Sinding, 2000) qui permettrait de prendre en compte le souhait des femmes.

La notion de besoins non satisfaits en contraception a été l'objet d'une pléthore de publications depuis les années 1970 (voir en particulier Westoff, 1978, 2006; Bongaarts, 1991, Govindasamy et Boadi, 2000; Mariko et al, 2009 ; Moreland Scott et Sharma, 2010). Les calculs ont été récemment affinés pour prendre en compte les femmes enceintes et allaitantes, les besoins des femmes non mariées, les échecs de contraception et les avortements (Bradley et al., 20I2 ${ }^{2}$ ). Définie comme la proportion de femmes qui déclarent ne pas souhaiter d'enfant, ou d'enfant supplémentaire, mais ne recourent pas à la contraception, la notion reste ancrée dans une vue de relation causale simple entre les intentions en matière de fécondité, l'utilisation de la contraception et la taille réalisée de la famille qui repose sur une série d'hypothèses discutables :

Le souhait d'enfant serait l'expression des intentions en matière de fécondité.

N'oublions pas qu'il s'agit là de ce que la femme estime approprié de déclarer au moment de l'enquête. Casterline et Layton (2013) soulignent que la femme n'exprime alors pas un besoin, mais ce que les auteurs interprètent comme une demande. Relative à la situation du moment, cette "demande" nous semble refléter non pas les intentions, mais les préférences (Andro et Hertrich, 200I) ou les convictions qui, comme le formule la Théorie du comportement planifié, constituent un préalable aux intentions ${ }^{3}$.

\section{L'offre créerait le besoin en contraception.}

Implicite est donc l'idée que la connaissance de la contraception et l'offre de services va créer et accroître le besoin d'y recourir et que ce besoin va se traduire en pratique. Cela implique que le fossé entre connaissance et pratique - largement connu dans la littérature sous le terme de KAP-Gap introduit par Mauldin (1965) - s'est estompé et donc que la connaissance entraîne une décision et la capacité d'utilisation.

L'accès à la contraception entrainerait systématiquement la baisse du niveau de fécondité.

La contraception serait alors une condition nécessaire et suffisante pour entraîner l'espacement et la limitation des naissances. On néglige donc les échecs contraceptifs et les autres stratégies de contrôle des naissances, y compris l'avortement. On aurait oublié, bien 
que souvent rappelé dans la littérature (p.ex. Pritchett, 1994), que la transition démographique en Europe a eu lieu bien avant l'ère de la contraception moderne et que presque toutes les sociétés dites traditionnelles ont été préoccupées par l'espacement des naissances (Bledsoe et al, 1998). On ignore les divers travaux qui montrent la relation faible entre l'accès à la contraception et le déclin de la fécondité (Pritchett, 1994) et, plus largement, entre celui-ci et les politiques de planning familial (Cosio-Zavala, 2006).

\section{Le modèle de la petite famille constituerait nécessairement un progrès.}

Finalement, implicite est l'idée d'un objectif universel de réduction de la taille de la famille. On néglige les raisons qui font que les gens souhaitent ou non un enfant à un certain moment et peuvent valoriser la grande famille. Comme formulé dans la théorie du Malthusianisme de la pauvreté (Cosio-Zavala, 200I), le déclin de la fécondité peut résulter de contraintes plus que de choix. De nombreuses raisons conduisent encore à valoriser un grand nombre d'enfants: stratégies de remplacement dans un contexte de maintien d'une mortalité infanto-juvénile élevée (LeGrand et al, 2003), contribution aux tâches familiales et diversification des stratégies de subsistance (Toulmin, 1992; Pilon and Vignikin, 1996), sécurité pour la vieillesse et part intégrante du statut des femmes et des hommes.

Certaines voix soulignent la nécessité de remplacer la vision centrée sur l'offre à une approche centrée sur la demande et les mécanismes de prise de décision (Casterline et Layton, à paraître) et plus largement d'adopter une approche fondée sur les droits qui considère les individus comme "agents actifs, et pas en tant que bénéficiaires passifs " (FNUAP, 20I2). Cela implique de s'interroger sur le bienfondé de l'hypothèse implicite derrière les quatre points cités ci-dessus, à savoir que les femmes sont en mesure de concevoir et réaliser un projet familial.

Nous postulons qu'un changement fondamental d'approche est nécessaire, c'est-àdire de mettre au centre le point de vue du sujet. Comme argumenté par Pike (Haedland et al, eds 1990), l'objectivation à travers des mesures qui font sens pour la communauté scientifique (l'approche étique), ne devrait être qu'un moyen pour appréhender ce qui fait sens pour la personne elle-même (point de vue émique). L'approche des Capabilités offre un cadre pertinent permettant de passer d'une analyse centrée sur le résultat (ici la pratique contraceptive et la fécondité atteinte) à une analyse des facteurs associés à la «liberté de réaliser ", c'est-à-dire ici d'explorer les facteurs qui permettent aux femmes de planifier et construire la famille qu'elles valorisent.

L'objectif de ce chapitre est donc de réanalyser les données EDS en appliquant le cadre des Capabilités. La prétention n'est pas de produire des résultats nouveaux, mais d'examiner dans quelle mesure le cadre des Capabilités permet de mieux comprendre les sources des inégalités dans la formulation et la réalisation des projets familiaux. II ne s'agit pas d'estimer la demande en contraception telle que développée dans l'approche fondée sur les besoins non satisfaits, mais de comprendre qui sont les couples qui utilisent la contraception et d'étudier le lien entre la pratique contraceptive et la constitution de la famille telle que mesurée par les intervalles entre les naissances. Cela implique de ré-examiner le fossé entre connaissance et pratique et identifier les autres facteurs influençant l'utilisation de la contraception. On s'interroge globalement sur la question de savoir si la contraception constitue une dimension de la liberté de planifier sa famille, c'est-à-dire une Capabilité dans le sens de Sen (voir les deux premiers articles de ce volume). Les préférences et choix individuels sont discutés dans l'article suivant sur la base des résultats d'une enquête qualitative.

\section{L'application du cadre des Capabilités: facteurs d'inégalités dans la maîtrise du projet familial}

Nous utilisons ici les données des enquêtes démographiques et de santé (EDS) réalisées au Mali en 2006 et au Ghana en 2008. L'échantillon du Mali est trois fois plus grand que celui du Ghana (14,583 et 4,916), ce qui permet donc des analyses plus poussées, en 
particulier la prise en compte du second intervalle intergénésique. Nous procédons en deux étapes: un premier " modèle » explore les sources d'inégalité d'accès à la contraception et un second l'association entre la contraception et l'espacement des naissances. Des régressions logistiques sur les Capabilités et les réalisations sont appliquées pour analyser les influences des caractéristiques utilisées comme indicateurs des ressources du contexte et des facteurs de conversion. Les corrélations entre ces différentes variables sont prises en compte par une procédure emboîtée qui introduit progressivement les différents blocs de variables.

\section{Modèle I : Analyse des facteurs de l'adoption d'une " culture contraceptive "}

Nous étudions ici l'hétérogénéité en matière de connaissance, d'utilisation et d'intentions de recourir à la contraception moderne. En d'autres termes, nous revisitons la question du fossé entre connaissance et pratique en examinant si la connaissance ouvre la voie vers la contraception, c'est-à-dire si elle peut être considérée comme une Capabilité. Est-ce que, plus qu'un moyen parmi d'autres, elle donne la capacité d'adopter la contraception. Dans ce but, nous analysons les facteurs d'inégalité de connaissance et quels facteurs d'hétérogénéité de pratique et d'intentions subsistent parmi ceux qui déclarent connaître les méthodes contraceptives.

Fig I. Facteurs de l'adoption d'une «culture contraceptive » Ressources du contexte Facteurs de Capabilités Préférences Accomplissement conversion

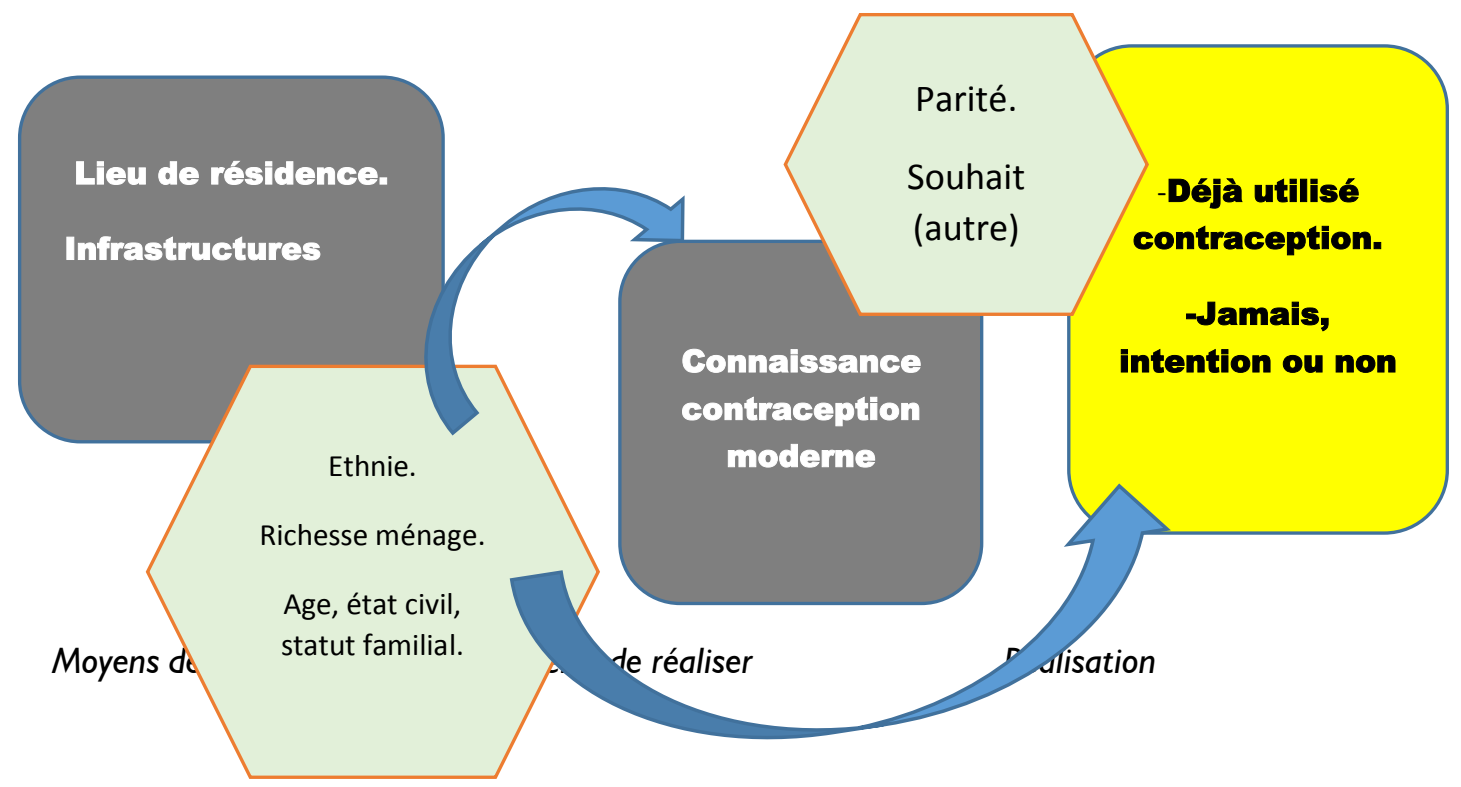

La Figure I représente la carte des variables, à savoir les indicateurs utilisés pour décrire les notions de base du cadre des Capabilités :

- Le lieu de résidence est utilisé comme indicateur de dotation collective à travers les différences en matière de ressources et infrastructures entre les grandes villes, les villes moyennes et les campagnes. L'enquête malienne fournit en plus des informations spécifiques sur la disponibilité d'infrastructures dans le voisinage.

- Pour les facteurs de conversion, on distingue les caractéristiques socio- 
culturelles telles que mesurées par l'appartenance ethnique, des spécificités individuelles données par la position dans le parcours de vie (l'âge), l'histoire et la situation familiale (âge au ${ }^{\mathrm{er}}$ mariage, état civil et statut dans le ménage, nombre d'enfants), ainsi que les ressources fournies par la richesse du ménage ${ }^{4}$, le niveau de scolarisation et le type d'occupation.
Modèle 2. Le rôle de la contraception dans la maîtrise du projet fécond.

Dans ce second modèle (Figure 2), nous examinons si l'adoption d'une "culture contraceptive » peut être considérée comme une Capabilité, c'est-à-dire une dimension de la capacité de maîtriser son projet familial. Nous classons les personnes selon trois niveaux de " culture contraceptive »: celles qui ont utilisé la contraception moderne au moins une fois dans leur vie et, parmi les autres, celles qui déclarent avoir ou non l'intention de le faire.

Fig. 2. Contraception et espacement des naissances

Capabilités Préférences

Facteurs de conversions
Accomplissement

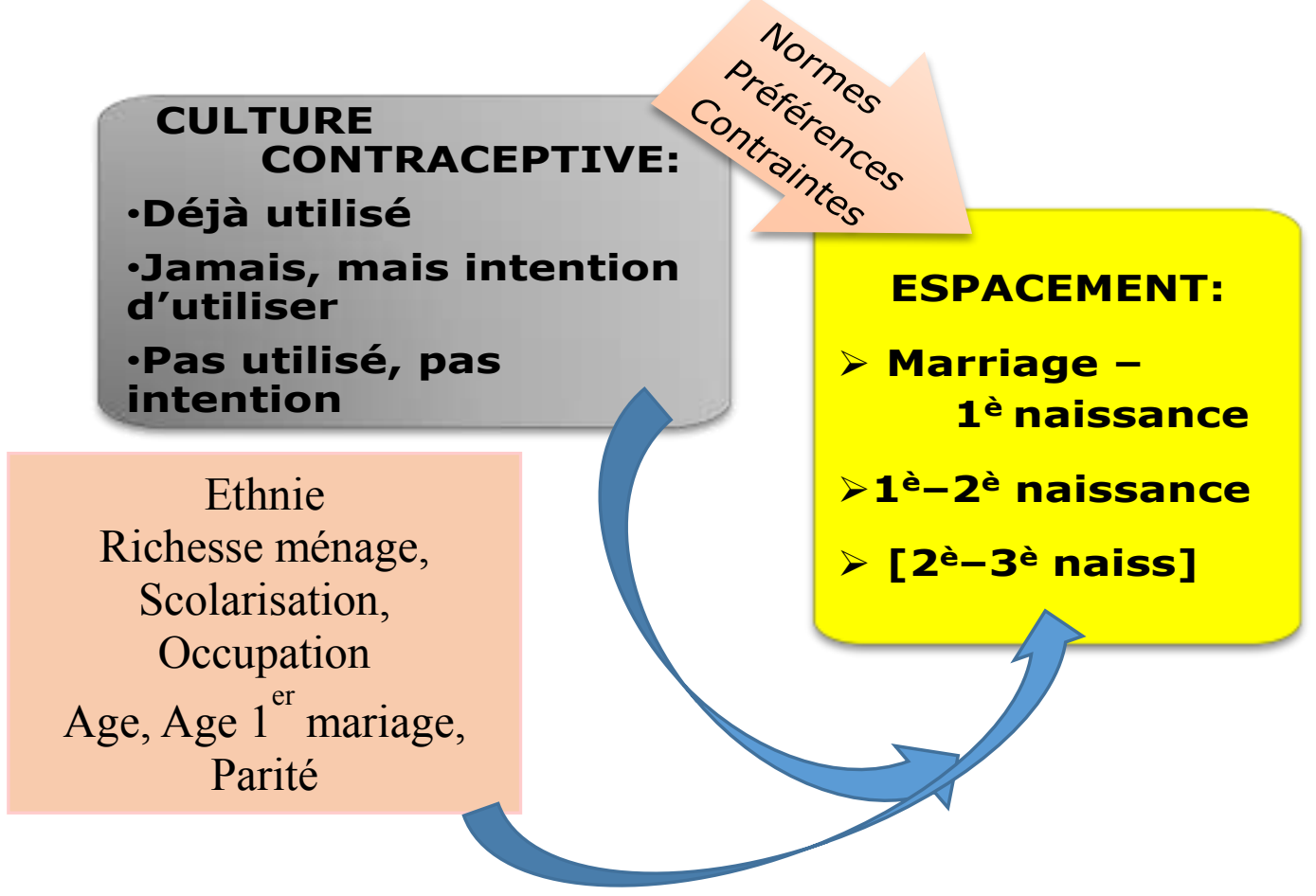


Nous examinons si ces différents niveaux de "culture contraceptive» se reflètent en matière de constitution de la famille, exprimée par l'espacement des naissances. Celui-ci, la résultante dans le schéma est mesuré par la probabilité d'appartenir au tiers supérieur de la distribution de la durée des intervalles protogénésiques (mariage-première naissance) et inter-génésiques (entre les naissances subséquentes). Nous postulons que, dans ce contexte, des longs intervalles traduisent une capacité de maîtrise du projet familial, reflétant des traditions d'espacement ou l'adoption de la contraception moderne. II convient aussi de garder à l'esprit la possibilité de l'émergence de nouveaux comportements de maternités rapprochées mais limitées.

Comme facteurs explicatifs, seules les caractéristiques constituant des indicateurs légitimes de la situation de l'individu au moment concerné (première, seconde et troisième naissances) ont été conservées. L'appartenance ethnique et la richesse du ménage ont été considérés comme suffisamment stables, mais le lieu de résidence, l'état civil et le statut dans la famille n'ont pas été retenus dans ce second modèle. La précocité de l'âge au mariage et le nombre d'enfants selon l'âge sont utilisés comme indicateurs d'adhésion à des valeurs familialistes de maximisation de la fécondité ${ }^{5}$ (tels que décrits par Locoh, 1995 ; Locoh et MouvaghaSow, 2005).

\section{Résultats}

\section{Adopter une "culture contraceptive" (Tableaux A et B en annexe)}

Le paradoxe entre une connaissance élevée de la contraception moderne et une pratique faible s'exprime non seulement en termes de fossé entre les niveaux de connaissance et de pratique, mais aussi dans l'hétérogénéité des facteurs qui influencent l'une et l'autre.

\section{a) Fossé entre connaissance et pratique : l'accès à l'information, une condition nécessaire mais pas suffisante pour adopter de nouveaux comportements.}

Dans les deux pays, le fossé entre connaissance et pratique reste énorme. Reflet des efforts importants de promotion du planning familial, la plupart des personnes interrogées déclarent qu'elles ont entendu parler des méthodes modernes de contraception: au Mali, c'est le cas de $70 \%$ des femmes et $87 \%$ des hommes, niveaux similaires aux résultats pour les zones rurales du Sénégal (Ndiaye et al, 2003). Au Ghana, la connaissance est presque universelle (respectivement $97 \%$ et $98 \%$ des réponses féminines et masculines), alors que l'utilisation de la contraception moderne reste limitée. Au Mali, seuls $7 \%$ des femmes et $6 \%$ des hommes déclarent utiliser présentement la contraception moderne et au Ghana, 17\% et 19\%. Cependant, dans les deux pays environ trois fois plus ont utilisé la contraception au moins une fois au cours de leur existence. Ceci confirme que le recours à la contraception est spécifique à certaines périodes de l'existence et que, par conséquent, la prévalence contraceptive, indicateur du moment retenu dans les OMD, n'a qu'un intérêt limité. II nous apparaît donc préférable de prendre en compte l'ensemble de l'histoire contraceptive, mesuré par l'utilisation de la contraception au moins une fois dans sa vie. Le fossé entre connaissance et pratique subsiste néanmoins. Parmi les personnes qui ont déclaré connaître la contraception moderne, au Mali à peine plus d'un quart ( $27 \%$ et $26 \%$ parmi les femmes et les hommes) et au Ghana un peu plus de la moitié $(51 \%$ et $56 \%)$ ont pratiqué la contraception. D'autre part, comme le montrent les analyses ci-après, des facteurs d'hétérogénéité d'utilisation subsistent parmi ceux et celles qui déclarent connaître la contraception.

\section{b) Les facteurs de l'adoption d'une " culture contraceptive ": hétérogénéité du niveau de connaissance et de pratique}

Par des analyses de régression logistique, nous examinons les facteurs associés à la 
connaissance, à l'utilisation et à l'intention d'utiliser la contraception moderne. Quels sont les facteurs qui accroissent à la fois la connaissance et la traduction de cette connaissance en pratique, quels facteurs ne modifient pas la pratique bien qu'ils accroissent la connaissance, ou qui sont importants pour l'adoption de la contraception, mais pas en matière de connaissance ${ }^{6}$ ?

Bien qu'élevé, le niveau de connaissance varie significativement selon les facteurs retenus ici. Surtout, la connaissance ne gomme pas les inégalités d'utilisation ou d'intention à utiliser.

Dans les deux pays, et pour les deux sexes, l'avantage brut, de connaissance et d'utilisation, lié à la résidence dans la capitale ou une grande ville disparaît lorsque l'on contrôle pour la corrélation avec l'effet des facteurs de conversion. Ce ne sont donc pas les conditions de vie des grandes villes qui font la différence, mais les capacités des citadins d'avoir accès aux connaissances et de les appliquer. Au Ghana, le lieu de résidence devient non significatif. Au Mali, l'effet de la connaissance et de la pratique devient significativement plus faible. Pour les femmes, résider dans une ville moyenne est un avantage à cet égard, tant par rapport aux grandes villes qu'aux zones rurales. Pour elles, la disponibilité en infrastructures (économiques, sanitaires et écoles) est cependant aussi un facteur décisif, ceci indépendamment du degré d'urbanisation ${ }^{7}$.

Dans les deux pays, l'adoption d'une " culture contraceptive " est influencée par l'appartenance ethnique même après contrôle pour le contexte de résidence et les caractéristiques personnelles et familiales. La distribution montre toutefois que ces différences socio-culturelles seraient renforcées par la localisation géographique. Au Mali, la connaissance est plus importante parmi les Bambaras, ethnie majoritaire et localisée autour de la capitale et, pour les hommes, parmi les groupes du sud, sud-ouest du pays (Senufo, Minyanka et Malinké) connus pour être en contact régulier avec des influences extérieures par leur proximité des frontières et par des traditions de migrations internationales. Elle est nettement moindre parmi les ethnies du Nord, ce qui est cohérent avec le fait qu'elles vivent dans des zones moins bien couvertes par les services. Ces mêmes disparités se retrouvent en matière d'utilisation, mais pour les femmes seulement. Pour les Touaregs, une forte autonomie résidentielle des femmes, rendrait la nécessité du recours à la contraception moins évidente. Au Ghana, les Akan et les Ewe ont une meilleure connaissance ce qui est consistant avec leur résidence dans le sud du pays, régions les plus modernisées. Cependant les Akans se distinguent par une pratique moindre qui reflète vraisemblablement leurs traditions matrilinéaires de séparation des résidences des époux.

La connaissance et la pratique est, dans les deux pays, moindre parmi les plus jeunes, ce qui confirme que les problèmes de manque d'adéquation des services aux besoins, en particulier en terme de confidentialité, n'ont pas été résolus. Au Mali, pour les jeunes femmes, le fait de ne pas être mariée apparaît comme un obstacle supplémentaire. Dans les deux pays, on retrouve aussi l'effet opposé bien connu entre cohorte et parité, à savoir un accroissement du recours à la contraception avec le nombre d'enfants, mais une culture contraceptive moindre parmi les cohortes plus anciennes. Cependant, dans les deux pays, le nombre d'enfants n'a aucun effet sur la pratique des hommes qui, en revanche, est associée à leur nombre idéal d'enfant. On constate aussi au Mali que, pour les hommes, le statut de chef de ménage n'est pas associé à une préoccupation de planning familial, au contraire la connaissance et la pratique est, toutes choses égales par ailleurs, plus faible pour eux. Au Ghana, si le statut de chef accroît la connaissance, il n'a pas d'effet sur l'utilisation.

Globalement, les ressources personnelles, toutes choses égales par ailleurs, accroissent la connaissance et la pratique. Ce n'est cependant que pour la scolarisation que l'effet est consistant pour les deux sexes. Pour tous, la richesse du ménage est associée à une meilleure pratique, bien qu'elle n'affecte pas le niveau de connaissance des hommes ghanéens. Globalement, on observe une meilleure acceptation des nouveaux comportements dans les secteurs moins traditionnels, c'est-à-dire non agricole et/ou non manuels, mais les effets sont complexes. Avoir une occupation non 
agricole accroît la pratique, mais l'effet sur la connaissance n'est significatif qu'au Mali et, pour les femmes, il n'améliore la connaissance que parmi celles ayant une activité non manuelle. D'autre part, si les femmes ne déclarant aucune activité professionnelle apparaissent avoir un handicap en termes de connaissance, en revanche, leur pratique est supérieure à celle des femmes actives dans le secteur agricole. Ainsi, travailler dans le secteur primaire n'apparaît pas associé à une ouverture sur le monde extérieur, généralement joué par l'insertion dans le marché du travail.

\section{c) Les intentions des femmes qui n'ont jamais utilisé la contraception mais pensent l'utiliser}

Environ un tiers (36\% au Mali, 39\% au Ghana) des femmes ${ }^{8}$, actives sexuellement qui déclarent connaître la contraception mais n'y ont jamais recouru, indiquent qu'elles ont l'intention de l'utiliser à l'avenir. Au Ghana, les intentions apparaissent moins influencées par les caractéristiques considérées que ce n'est le cas pour l'utilisation. Au Mali, la plupart des effets subsistent mais pas toujours dans le même sens.

Certaines caractéristiques influencent dans le même sens la pratique et les intentions futures. Ainsi, au Mali, les deux sont moindres parmi les femmes qui résident dans une grande ville, sans infrastructures économiques à proximité, qui appartiennent à des ethnies du nord du pays (Soninke/Songhai/Tamasheq) et aux cohortes de plus de 30 ans. Elles sont accrues par le nombre d'enfants et la scolarisation. Au Ghana, on retrouve les mêmes influences du nombre d'enfants et de la cohorte.

Certains groupes de femmes qui, pour des raisons variées, n'ont pas encore utilisé la contraception, se distinguent par des intentions accrues. C'est le cas des plus jeunes et de celles qui travaillent dans le secteur agricole et, au Mali, aussi celles qui vivent dans les zones sans écoles ou structures de santé. Au Ghana, le désavantage, en termes d'utilisation, des femmes non scolarisées et/ou qui vivent dans les ménages les plus pauvres disparaît en ce qui concerne les intentions.

Finalement, un résultat important est que, parmi les femmes au Ghana et parmi les hommes dans les deux pays, l'adoption d'une culture contraceptive telle qu'exprimée par la pratique et les intentions n'apparait pas totalement cohérente avec les souhaits d'enfants. Certes, les femmes qui déclarent souhaiter un enfant dans les deux prochaines années ont une culture contraceptive moindre (elles ont moins souvent déjà eu recours à la contraception ou ont moins l'intention d'y recourir) que celles qui préfèrent attendre. Alors qu'au Mali celles qui disent ne plus souhaiter d'enfants ont plus souvent déjà utilisé la contraception, au Ghana, au contraire elles l'ont moins pratiquée. Dans les deux pays, la pratique des hommes n'est pas associée à leurs déclarations de souhaits d'enfants.

\section{Rôle de la contraception dans la maîtrise du projet fécond (Tableaux C et D en annexe)}

Dans les deux pays, l'intervalle protogénésique est beaucoup plus court que les intervalles entre les naissances: deux-tiers des femmes ont leur premier enfant moins de deux ans après le mariage, alors que deux-tiers attendent plus de deux ans pour avoir le second au Mali et, au Ghana, un-tiers retarde la seconde maternité au-delà de 3 ans et demi (Tableau I).

\section{Tableau I. Durée des intervalles entre les naissances}

\begin{tabular}{|c|c|c|c|c|c|c|}
\hline $\begin{array}{l}\text { INTERVALLES } \\
\text { (en mois) }\end{array}$ & $\begin{array}{c}\text { Mali } \\
\text { Jer } \\
\text { tercile }\end{array}$ & $\begin{array}{r}2^{\mathrm{è}} \\
\text { tercile }\end{array}$ & $\begin{array}{r}3^{\grave{e}} \\
\text { tercile }\end{array}$ & $\begin{array}{c}\text { Ghana } \\
\qquad\left.\right|^{\mathrm{er}} \\
\text { tercile }\end{array}$ & $\begin{array}{r}2^{\mathrm{è}} \\
\text { tercile }\end{array}$ & $\begin{array}{r}3^{\grave{e}} \\
\text { tercile }\end{array}$ \\
\hline Marriage-Iè naissance & $0-12$ & $13-26$ & $27+$ & $0-11$ & $12-23$ & $24+$ \\
\hline lè - 2è naissances & $0-23$ & $24-32$ & $33+$ & $0-28$ & $29-44$ & $45+$ \\
\hline 2è - 3è naissances & $0-23$ & $24-32$ & $33+$ & -- & & \\
\hline
\end{tabular}


Dans les deux pays, l'intervalle protogénésique est non seulement plus court, mais il n'est pas influencé par l'adoption d'une culture contraceptive (Tableau C en annexe). En revanche, les intervalles inter-génésiques apparaissent plus longs parmi les femmes qui ont adopté la contraception. Cet effet de la «culture contraceptive" subsiste lorsque l'on contrôle pour les caractéristiques culturelles telles qu'exprimées par l'appartenance ethnique, mais il s'estompe avec la prise en compte des caractéristiques personnelles (et, au Mali, disparaît même pour le second intervalle). Ainsi, plus que l'adoption de la contraception, ce sont les caractéristiques de celles qui l'on adopté qui comptent ${ }^{\text {ix. }}$.

L'examen de la diversité met en évidence un antagonisme entre traditions et modernité. D'une part, dans les deux pays, subsistent des particularités liées à l'appartenance ethnique, même en contrôlant pour le contexte de résidence et les ressources personnelles. Ainsi les Mole-Dagbani au Ghana, connus pour être plus traditionnels se distinguent par le maintien de longs intervalles tant proto- que intergénésiques. II en est de même au Mali pour les ethnies du Sud (catégorie autres) qui donc auraient des traditions d'espacement au début de la vie familiale. L'incidence moindre des longs intervalles parmi les Bambaras ${ }^{x}$, comme aussi parmi les cohortes les plus récentes, témoigne apparemment d'un effet de modernisation.

La variabilité due aux caractéristiques personnelles souligne la montée de «l'Afrique des individus» (Marie, ed 1997). Les effets des ressources personnelles sont plus clairs sur les intervalles inter- que proto-génésiques, particulièrement au Mali. Ceci est consistent avec un espace de liberté réduit en matière de calendrier de la première naissance. L'accroissement des ressources n'a toutefois pas un effet uniforme sur la durée des intervalles.

Au Mali, les plus riches et les plus scolarisées ont de plus longs intervalles intergénésiques. Cependant, être active dans le secteur agricole (ou, pour le second intervalle inter-génésique, avoir une autre activité manuelle) accroît aussi la durée des intervalles, ce qui peut exprimer la persistance de traditions d'espacement. On aurait donc affaire à des effets complexes de diffusion de nouveaux comportements, de maintien de traditions et de contraintes de la pauvreté. C'est aussi ce qui est illustré par la forme en $U$ de l'effet de la richesse du ménage sur l'intervalle proto-génésique: ce sont à la fois les plus pauvres et les plus riches qui repoussent la première naissance. La thèse de deux types opposés de déterminants de la diminution de la taille de la famille - qualité de vie et statut de la femme, mais aussi contraintes de la pauvreté (Malthusianisme de la pauvreté : Cosio-Zavala, 200I) - doit donc être complétée par une troisième dimension qui est celle de la persistance de traditions d'espacement.

Au Ghana, globalement, les ressources personnelles ont peu d'influence sur l'espacement des naissances. La richesse du ménage, le niveau de scolarisation et le type d'occupation n'influencent pas l'intervalle proto-génésique et les deux premiers perdent leur significativité lorsque l'on contrôle pour le familialisme. Comme au Mali les femmes actives dans des professions manuelles sont plus souvent dans le groupe de celles qui retardent la venue du second enfant.

Pour les deux pays, dans tous nos modèles, l'adhésion à des valeurs familialistes, exprimée par la taille de la famillexi, est associée à des intervalles plus courts. En revanche, un âge au mariage très précoce (avant 15 ans) est associé à un report de la première naissance et des intervalles plus longs qui exprimeraient des préoccupations relatives aux risques liés aux maternités trop précoces. Un mariage tardif, en revanche, entraîne un rattrapage à savoir une première naissance plus rapidement après le mariage.

\section{Conclusions}

La question centrale est ici de savoir si la contraception représente une Capabilité, à savoir une dimension de la liberté de réaliser son projet familial. En mettant l'accent sur les processus comportementaux plutôt que les résultats atteints, l'approche des Capabilités amène à examiner le planning familial en termes d'adoption d'une culture contraceptive 
et de son rôle dans la maîtrise du projet d'enfant.

\section{Les facteurs associés à l'adoption d'une culture contraceptive}

Les résultats confirment que le recours à la contraception est fortement dépendant de la position dans le parcours de vie familiale et que, par conséquent, l'image du moment fournie par la prévalence contraceptive ne constitue pas un reflet fidèle du comportement contraceptif. D'autre part, la connaissance de la contraception ne gomme pas les inégalités en matière de pratique. Le fossé entre connaissance et pratique reste très large, et de fortes inégalités de pratique subsistent parmi ceux qui déclarent connaître les méthodes modernes de contraception. Les facteurs accroissant la connaissance ne sont pas forcément les mêmes que ceux qui amènent les individus à adopter une culture contraceptive, ou encore certains facteurs accroissent la pratique mais n'ont pas d'effet sur la connaissance. Ainsi la connaissance est une condition nécessaire mais pas suffisante pour recourir à la contraception. C'est donc une approche plus large d'adoption d'une culture contraceptive que nous avons retenue en examinant les facteurs influençant la connaissance et, parmi ceux qui déclarent connaître, l'utilisation ou l'intention d'utiliser.

\section{a) Les facteurs complexes d'utilisation des ressources en matière d'information et d'offre de planification familiale}

La variabilité en matière de connaissances et de pratique est expliquée dans une certaine mesure par les caractéristiques du lieu de résidence et de la région d'appartenance. Toutefois, les résultats mettent bien en évidence que subsistent des différences entre individus résultant de leurs "facteurs de conversion", mesurés par leurs caractéristiques personnelles et leur l'ancrage culturel. L'avantage des habitants des grandes villes, en termes de connaissance et d'utilisation accrues, disparaît lorsque l'on contrôle pour les caractéristiques personnelles: celles-ci modulent donc des inégalités d'accès aux possibilités offertes par les grandes villes.
L'hétérogénéité ethnique exprime la persistance de spécificités culturelles définissant des bassins géographiques dépassant la distinction urbain/rural Ainsi les populations appartenant à la majorité représentée dans le périmètre de la capitale ont une culture contraceptive plus élevée et, au Mali, celle-ci est spécialement basse parmi les ethnies du Nord où le développement économique a été le plus faible.

Dans les deux pays, les plus jeunes apparaissent désavantagés tant en matière de connaissance que d'utilisation et, au Mali, encore plus s'ils sont célibataires. Ceci reflète le problème souvent cité d'inadéquation des services pour répondre aux besoins des jeunes et du maintien, au Mali, d'une discrimination envers les personnes non mariées.

b) Inégalités entre individus : les ressources personnelles et le genre

Les ressources personnelles - mesurées ici par le fait de vivre dans les ménages plus riches, d'avoir fait des études et d'avoir une profession non manuelle (non agricole pour les hommes) constituent un élément central des différences de connaissance et de pratique pour les deux sexes dans les deux pays. Ce rôle clé des ressources personnelles souligne bien l'importance de la dimension individuelle de l'adoption d'une culture contraceptive. En revanche, les ressources personnelles apparaissent moins importantes dans le différentiel en matière d'intentions.

Les différences de genre sont cohérentes, spécialement au Mali, avec une plus grande dépendance des femmes par rapport à la réalité de leur situation et de leur contexte de vie.

D'une part, au Mali, les hommes semblent moins influencés par les caractéristiques de leur contexte de vie. D'autre part, dans les deux pays, femmes et hommes ne sont pas influencés de la même façon par leur statut dans la famille et leur nombre d'enfants. Pour les hommes, particulièrement au Mali, être chef de famille n'est pas associé à des préoccupations accrues en matière de planning familial, au contraire, leur connaissance et pratique de la contraception sont moindres. Pour les femmes, le statut de cheffe de ménage 
n'a pas d'effet sur le recours à la contraception, bien que, au Ghana, il accroisse la connaissance. Ainsi, être cheffe de ménage ne semble pas leur donner plus de capacité de décision. Surtout, alors que dans les deux pays, pour les deux sexes, l'adoption d'une culture contraceptive est inversement proportionnelle au nombre idéal d'enfants, en revanche ce n'est que pour les femmes qu'elle est aussi associée à la réalité familiale, mesurée par le nombre d'enfants et le souhait d'enfant supplémentaire. Ainsi, pour les hommes, l'adoption d'une culture contraceptive ne semble pas être liée aux contingences du quotidien mais à des valeurs familiales. Ceci est cohérent avec les commentaires des femmes dans l'enquête qualitative sur le fait que ce sont elles qui assument les charges familiales quotidiennes.

\section{Le rôle de la culture contraceptive dans} la maîtrise du projet fécond

La présente analyse est fondée sur le postulat que, dans le contexte ouest-africain, l'adoption de la contraception et son rôle dans la baisse de la fécondité sont rendus complexes par le fait que les femmes ont rarement un projet fécond préconçu et que, plutôt, elles s'adapteraient et réagiraient aux situations réelles qu'elles vivent. Ceci se reflète notamment dans la prévalence plus élevée de contraception d'espacement que de limitation. Nous considérons donc ici la maîtrise du projet fécond à travers la durée des intervalles entre les naissances.

Les résultats témoignent d'une situation de transition entre d'anciennes traditions d'espacement ancrées dans des valeurs collectives et des comportements plus individuels. C'est ainsi que l'on observe l'effet de facteurs de nature culturelle et d'adhésion à des valeurs familialistes, mais aussi de dimensions plus individuelles révélées par les différences liées aux ressources personnelles. Les traditions apparaissent plus prégnantes en matière d'intervalle entre le mariage et la première naissance que pour les intervalles entre les naissances. Au Ghana, les ressources personnelles n'ont pas d'effet sur l'intervalle proto-génésique, ce qui peut être interprété comme une faible marge de manœuvre individuelle à cet égard. Au Mali, leur effet est complexe. Ce sont les femmes avec moins de ressources qui repoussent la venue du premier enfant, ce qui peut exprimer des obstacles liés à la pauvreté ou un lien entre un faible niveau de ressources et des valeurs plus traditionnelles.

En revanche, les ressources personnelles apparaissent plus importantes pour déterminer les différences en matière d'intervalles intergénésiques. Les facteurs culturels ne sont cependant pas absents comme en témoignent la variabilité selon l'ethnie, les plus longs intervalles parmi les cohortes plus anciennes et, au Mali pour le second intervalle, parmi les femmes actives dans l'agriculture. II semblerait donc que, parmi les secteurs plus en contact avec de nouvelles valeurs, émerge un comportement de concentration des naissances en début de vie conjugale.

La culture contraceptive n'apparaît ici que faiblement associée à l'espacement des naissances. Elle n'a aucun effet sur l'intervalle proto-génésique et, lorsque l'on contrôle pour les caractéristiques personnelles, son influence sur le premier intervalle inter-génésique diminue et, au Mali, il disparaît sur le second. Ainsi ce n'est pas l'adoption de la contraception qui importe, mais le profil des femmes qui l'adoptent.

La contraception, en soi, ne représente pas une transformation des valeurs, mais constitue plutôt un moyen pour réaliser des intentions influencées par d'autres facteurs. Par conséquent, le manque d'accès à des services de qualité peut être un obstacle, mais ne détermine pas les décisions en matière de fécondité. La contraception n'a qu'un impact limité sur la maîtrise du projet familial, elle ne constitue donc pas une Capabilité, mais un moyen parmi d'autres pour espacer les naissances. Ceci est cohérent avec le déclin marqué de la fécondité au Ghana, en dépit d'une prévalence contraceptive relativement faible.

Par l'analyse des résultats d'une enquête qualitative, nous explorons dans l'article suivant la marge de liberté dont disposent les femmes pour gérer leur projet familial. Ce qui reste à mieux comprendre c'est le rôle que jouent 
d'autres facteurs tels, au Ghana, les coutumes de résidence séparée des époux (Hill et al, sous presse), sujet qui dépasse l'objectif du présent travail.

\section{Références}

Ajzen, I. (1988), Attitudes, Personality and Behavior, Open University Press, Milton Keynes.

Andro, Armelle et Véronique Hertrich (200I), La demande contraceptive au Sahel: les attentes des hommes se rapprochent-elles de celles de leurs épouses? Population 56(5), 72I-77I.

Billari, Francesco, Dimiter Philipov, Maria R. Testa (2009), Attitudes, norms and perceived behavioural control: Explaining fertility intentions in Bulgaria. European Journal of Population, 25(4), 439-465.

Bledsoe, Caroline, Fatou Banja and Allan G. Hill (1998), Reproductive mishaps and western contraception: An African challenge to fertility theory. Population and Development Review, 24(I), 15-57.

Bongaarts, John (I99I), The KAP-gap and the unmet need for contraception. Population and Development Review, I7(2), 293-313.

Casterline, John and Sarah Layton (20/3), Unmet need and its causes: A literature Review. Seminar 'Is access enough? Understanding and addressing unmet need in Family Planning', IUSSP Scientific Panel Reproductive Health, Kenya.

Casterline, John and Steven Sinding (2000), Unmet Need for Family Planning in Developing Countries and Implications for Population Policy. Population and Development Review, 26 (4), 69I-723.

Cohen, Susan (2012), London Summit Puts Family Planning Back on the Agenda, Offers New Lease on Life for Millions of Women and Girls.

http://www.guttmacher.org/pubs/gpr/I5/3/gpr I 50320.pdf

Cosio-Zavala, Maria Eugenia (200I), Les deux modèles de transition démographique en Amérique latine: le malthusianisme de la pauvreté. In Transitions Démographiques des Pays du Sud, Gendreau F. et $M$. Poupard (eds), Editions Estem, Paris, 4l-52.
Cosio-Zavala, Maria Eugenia (2006), Politiques de population au Mexique. In Caselli, Vallin et Wünsch, Histoire des idées et politiques de population. INED, Paris, 625-646.

FNUAP (20I2), Oui au choix, Non au hasard. Planification familiale, droits de la personne et développement. Etat de la population mondiale, New York.

Govindasamy, Pav and Emmanuel Boadi (2000), A decade of unmet need for contraception in Ghana: Programmatic and policy implications. Calverton, Maryland: Macro International.

Headland, Th.N., K.L. Pike and M. Harris (eds, 1990), Emics and Etics. The Insider/Outsider Debate. Frontier of Anthropology, V. 7. Summer Inst. of Linguistics, Sage.

Hill, Allan G., Samuel Agyei-Mensah and John K. Anarfi (In Press): Towards Replacement Level Fertility in Urban Africa: An Accra Case Study. Chapter II, African Population Studies.

LeGrand, Thomas, Todd Koppenhaver, Nathalie Mondain, Dominique Gomis, Sara Randall and Kenneth Hill (2003), Reassessing the insurance effect: A qualitative analysis of fertility behaviour in Senegal and Zimbabwe. Population and Development Review, 29(3), 375-403.

Locoh, Thérèse (1995), Familles africaines, population et qualité de vie. Dossiers CEPED 31.

Locoh, Thérèse et Myriam Mouvagha-Sow (2005), Vers de nouveaux modèles familiaux en Afrique de l'Ouest? XXVè Congrès international UIESP, Tours.

Marie, Alain (ed, 1997), L'Afrique des individus, Itinéraires citadins dans I'Afrique contemporaine. Paris, Karthala.

Mariko, Soumaila, Mohamed Ayad, Rathavuth Hong, Oumou Kéïta et Mamadou Diop (2009), Pratique contraceptive et importance des besoins non satisfaits en matière de planification familiale au Mali, de 1995 à 2006: Analyses approfondies des Enquêtes Démographiques et de Santé au Mali. Calverton, USA. 
Mauldin, W. Parker (1965), Fertility Studies: Knowledge, attitude and practice. Studies in Family Planning I (7), I-I0.

Moreland, Scott, Ellen Smith and Suneeta Sharma (2010), World Population Prospects and Unmet Need for Family Planning. Futures Group and Hewlett Foundation.

Ndiaye, C.A.T., V. Delaunay et A. Adjamagbo (2003), Connaissance et utilisation des méthodes contraceptives en milieu rural sereer au Sénégal. Cahiers Santé, I 3 (I), 3137.

Pilon Marc et Kokou Vignikin (1996), Stratégies face à la crise et changements dans les structures familiales. Chap. 18 in Coussy et Vallin (eds), Crise et population en Afrique. Etudes CEPED 13.

Pritchett, Lant H. (1994), Desired Fertility and the Impact of Population Policies. Population and Development Review 20(I), I-55.

Rutstein, Shea O. (20II), Trends in Birth Spacing. DHS Comparative Reports 28. Calverton, USA.

Rutstein, Shea O. and Kiersten Johnson (2004), The DHS Wealth Index. DHS Comparative Reports No 6. Calverton, USA.
Sek, Bradley, T.N. Croft, J.D. Fishel and C.F.Westoff (20I2), Revising unmet need for family planning. DHS Analytical Studies 25. Calverton: ICF International.

Toulmin, Camilla (1992): Cattle, women and wells. Oxford.

Ushma, D., B. Upadhyaya and M. J. Hindin (2005), Do higher status and more autonomous women have longer birth intervals? Results from Cebu, Philippines. Social Science \& Medicine 60, 264 I-2655.

Westoff, Charles (1978), The Unmet Need for Birth Control in Five Asian Countries. Family Planning Perspective 10 (3), I73I8I.

Westoff, Charles (2006), New Estimates of Unmet Need and the Demand for Family Planning. DHS Comparative Report 14, Macro International, Calverton, USA.

Yamin, Alicia Ely and Vanessa Boulanger (2013), Embedding sexual and reproductive health and rights in a transformational development framework: lessons learned from the MDG targets and indicators. Reproductive Health Matters. 
Annexe. Tabl. A. Facteurs associés à la connaissance, l'utilisation et les intentions d'utiliser la contraception moderne au Mali (EDS 2006). Régression logistique par blocs (nested): $\beta_{0}+\beta_{1} x_{1}+\ldots+\beta_{n} x_{n}=\ln (P x / I-P x)$. Valeurs fournies : odd ratio (Exp du coefficient $\beta$ )

\begin{tabular}{|c|c|c|c|c|c|c|c|c|c|}
\hline & & \multicolumn{8}{|c|}{ MALI - FEMMES } \\
\hline \multirow{16}{*}{ 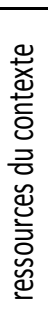 } & \multirow{4}{*}{$\begin{array}{|rr|}\text { Résidence } & \text { capitale, grande vill } \\
\text { petite ville } \\
\text { campagnes }\end{array}$} & \multicolumn{2}{|c|}{ CONNAISSANCE } & & \\
\hline & & $2.21^{* * *}$ & $0.80 *$ & \multicolumn{3}{|c|}{$1.18^{* *} \quad 0.71^{* * *} 0.72^{* * *}$} & \multicolumn{3}{|c|}{ INTENTION } \\
\hline & & 1.00 & 1.00 & 1.00 & 1.00 & 1.00 & 1.00 & 1.00 & 1.00 \\
\hline & & $0.76 * * *$ & $0.84 * * *$ & $0.52 * * *$ & $0.78 * * *$ & $0.78^{* * *}$ & $1.26 * * *$ & 1.11 & 1.12 \\
\hline & Infrastructures économiques (nb) & $0.65^{* * *}$ & $0.74 * * *$ & $0.83 * *$ & $0.86 *$ & $0.86 *$ & $0.62^{* * *}$ & $0.70^{* * *}$ & $0.70^{* * *}$ \\
\hline & $1-3$ & 1.00 & 1.00 & 1.00 & 1.00 & 1.00 & 1.00 & 1.00 & 1.00 \\
\hline & 4-5 & 1.02 & 1.10 & 0.90 & 0.94 & 0.94 & $1.25 * *$ & $1.25 * *$ & $1.21 *$ \\
\hline & Ecole & $1.31^{* * *}$ & $1.26 * * *$ & $1.16 *$ & 1.11 & 1.13 & $1.17^{* *}$ & $1.23 * * *$ & $1.24^{* * *}$ \\
\hline & non & 1.00 & 1.00 & 1.00 & 1.00 & 1.00 & 1.00 & 1.00 & 1.00 \\
\hline & Station de radio & 1.05 & 0.99 & $1.22 * * *$ & $1.16^{* *}$ & $1.16^{* *}$ & 0.93 & 0.92 & 0.93 \\
\hline & 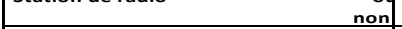 & 1.00 & 1.00 & 1.00 & 1.00 & 1.00 & & 1.00 & 1.00 \\
\hline & Services de santé & $0.67^{* * *}$ & $0.70^{* * *}$ & $0.82^{* * *}$ & 0.95 & 0.96 & $1.26^{* * *}$ & $1.21^{* *}$ & $1.22 * * *$ \\
\hline & 1-2] & 1.00 & 1.00 & 1.00 & 1.00 & 1.00 & 1.00 & 1.00 & 1.00 \\
\hline & $3+$ & $1.60^{* * *}$ & $1.26 * *$ & $1.29^{* * *}$ & $1.24 * *$ & $1.23 * *$ & 1.08 & 1.00 & 0.99 \\
\hline & Pharmacie & 1.07 & 1.04 & $1.20^{* * *}$ & $1.14 *$ & $1.15^{* *}$ & 1.03 & 1.11 & 1.13 \\
\hline & nol & 1.00 & 1.00 & 1.00 & 1.00 & 1.00 & 1.00 & 1.00 & 1.00 \\
\hline \multirow{33}{*}{ 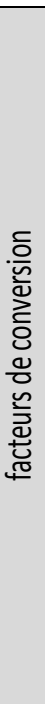 } & Bambara & & 1.00 & & 1.00 & 1.00 & & 1.00 & 1.00 \\
\hline & Malinké| & & $0.82 *$ & & 1.16 & 1.19 * & & 0.92 & 0.93 \\
\hline & Peulh & & $0.42 * * *$ & & 0.88 & 0.91 & & $0.64 * * *$ & $0.65 * * *$ \\
\hline & Sarkolé/Soninké/Marka & & $0.32 * * *$ & & $0.83 * *$ & $0.84 *$ & & $0.60^{* * *}$ & $0.60 * * *$ \\
\hline & Sonrai & & $0.14 * * *$ & & $0.60 * * *$ & $0.65 * * *$ & & $0.27 * * *$ & $0.31 * * *$ \\
\hline & Dogon & & $0.17 * * *$ & & 0.98 & 1.06 & & $0.38 * * *$ & $0.42 * * *$ \\
\hline & Touareg & & $0.26 * * *$ & & $0.75 * *$ & $0.73^{* *}$ & & $0.25 * * *$ & $0.22 * * *$ \\
\hline & Sénoufo/Minianka & & $0.56 * * *$ & & 0.92 & 0.93 & & 1.02 & 1.05 \\
\hline & Bobo/Autre & & $0.31 * * *$ & & 0.94 & 0.97 & & $0.56 * * *$ & $0.57^{* * *}$ \\
\hline & Richesse ménage (quintile) plus pauvre & & 0.99 & & 0.86 & 0.88 & & 1.08 & 1.14 \\
\hline & Richesse menage (quintile) $\begin{array}{r}\text { plus paure } \\
\text { pauvre }\end{array}$ & & 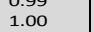 & & 0.95 & 0.96 & & 1.09 & 1.15 \\
\hline & $\begin{array}{c}\text { pauvre } \\
\text { moyen }\end{array}$ & & 1.00 & & $\begin{array}{l}.95 \\
1.00\end{array}$ & 1.00 & & 1.00 & 1.00 \\
\hline & riche & & $1.22 * * *$ & & $1.26 * * *$ & $1.26 * * *$ & & 0.89 & 0.91 \\
\hline & $\begin{array}{l}\text { riche } \\
\text { plus riche }\end{array}$ & & $2.68 * * *$ & & $1.98 * * *$ & $2.01 * * *$ & & 1.11 & $\begin{array}{l}.91 \\
1.14\end{array}$ \\
\hline & Groupe d'âge & & $0.52^{* * *}$ & & $0.46^{* * * *}$ & $\frac{2.01}{0.48^{* * *}}$ & & $1.61^{* * *}$ & $1.63 * * *$ \\
\hline & $\begin{array}{r}15-199 \\
20-29\end{array}$ & & 1.00 & & 1.00 & 1.00 & & 1.00 & 1.00 \\
\hline & 30-39 & & $0.91 *$ & & $1.15 * *$ & 0.87 * & & $0.56 * * *$ & $0.57 * * *$ \\
\hline & $40-49$ & & $0.59 * * *$ & & & $0.61 * * *$ & & $0.14^{* * *}$ & $0.13 * * *$ \\
\hline & $\begin{array}{r}50-59 \\
\text { marié(e)/cohabitant(e) }\end{array}$ & & $2.16^{* *}$ & & $1.40^{* * *}$ & $1.46 *$ & & 0.99 & 1.0 \\
\hline & [élibataire/divorcé(e)/veuve-veuf| & & 1.00 & & 1.00 & 1.00 & & 1.00 & 1.00 \\
\hline & tatut dans ménage & & 1.16 & & 0.94 & 0.93 & & $0.44^{* * *}$ & $0.44^{* * *}$ \\
\hline & époux/se & & 1.03 & & 0.94 & 0.88 & & $0.82 * *$ & $0.81 * *$ \\
\hline & autre & & 1.00 & & 1.00 & 1.00 & & 1.00 & \\
\hline & Scolarisation & & $0.52 *$ & & $0.52 * * *$ & $0.52 * * *$ & & $0.81 * *$ & $0.81 * *$ \\
\hline & $\begin{array}{l}\text { coliarisation } \\
\text { primaire (qques années ou achevé) }\end{array}$ & & 1.00 & & 1.00 & 1.00 & & 1.00 & 1.00 \\
\hline & 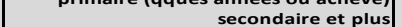 & & 2.67 & & 1.18 & 1.18 * & & 1.23 & 1.18 \\
\hline & Activité prof & & $0.74^{* * *}$ & & $1.19 * *$ & $1.13^{* * *}$ & & $0.71^{* * *}$ & $0.73^{* * *}$ \\
\hline & non-manuelle & & $1.68 * * *$ & & $1.65 * * *$ & $1.72 * * *$ & & 1.08 & 1.12 \\
\hline & agriculture & & 1.00 & & 1.00 & 1.00 & & 1.00 & 1.00 \\
\hline & manuelle & & & & $1.46 * * *$ & $1.61 * * *$ & & $0.78 * *$ & 0.83 \\
\hline & Nb enfants vivants & & & & & $\frac{1.01}{1.00}$ & & 1.00 & 1.00 \\
\hline & 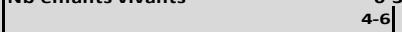 & & & & & $1.49 * * *$ & & $1.65 * * *$ & $1.49^{* * *}$ \\
\hline & & & & & & $1.6 \mathrm{~s}$ ( 10 & & $2.88 * * *$ & $2.37 * * *$ \\
\hline \multirow{5}{*}{ 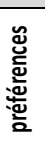 } & Souhait enfant suppl & & & & & $1.32^{* * *}$ & & & \\
\hline & dans les 2 ans & & & & & $0.68 * * *$ & & & $0.48^{* * *}$ \\
\hline & au-delà de 2 ans & & & & & 1.00 & & & 1.00 \\
\hline & Nb idéal d'enfants & & & & & & & & \\
\hline & $4-6$ & & & & & 1.00 & & & 1.00 \\
\hline
\end{tabular}

\begin{tabular}{|c|c|c|c|}
\hline \multicolumn{4}{|c|}{ MALI - HOMMES } \\
\hline \multicolumn{2}{|c|}{ CONNAISSANCE } & \multicolumn{2}{|c|}{ UTILISE AU MOINS UNE FOIS } \\
\hline $1.76 * *$ & $0.44^{* *}$ & $2.03^{* * *}$ & 0.96 \\
\hline & 1.00 & & 1.00 \\
\hline $0.68 * *$ & & $0.50^{* * *}$ & \\
\hline $0.48^{* * *}$ & $0.64 * * *$ & 0.80 & 0.91 \\
\hline 1.00 & 1.00 & & 1.00 \\
\hline 1.29 & 1.53 & 1.16 & \\
\hline 1.21 & 1.04 & $1.69^{* * *}$ & $1.68^{* * *}$ \\
\hline 1.00 & 1.00 & & \\
\hline 1.12 & 0.96 & $1.33^{* *}$ & 1.19 \\
\hline 1.00 & 1.00 & 1.00 & 1.00 \\
\hline $0.61^{* * *}$ & $0.61 * * *$ & 0.84 & 0.95 \\
\hline 1.00 & 1.00 & 1.00 & 1.00 \\
\hline 1.69 & 1.47 & 0.79 & $0.72 * *$ \\
\hline 0.86 & 0.76 & 1.16 & 1.00 \\
\hline \multirow[t]{40}{*}{1.00} & 1.00 & 1.00 & 1.00 \\
\hline & 1.00 & & 1.00 \\
\hline & $1.60 *$ & & 1.09 \\
\hline & $0.41 * * *$ & & 1.01 \\
\hline & 0.98 & & 0.83 \\
\hline & $0.39 * * *$ & & 1.20 \\
\hline & $0.39 * * *$ & & 1.02 \\
\hline & $0.17^{* * *}$ & & $0.56 *$ \\
\hline & $1.87^{* *}$ & & 1.31 \\
\hline & $0.49 * * *$ & & 0.83 \\
\hline & 0.82 & & 0.85 \\
\hline & 1.09 & & 0.78 \\
\hline & 1.00 & & 1.00 \\
\hline & 0.87 & & 1.04 \\
\hline & $2.69 * * *$ & & $2.18 * * *$ \\
\hline & $0.29^{* * *}$ & & 0.77 \\
\hline & 1.00 & & 1.00 \\
\hline & 1.15 & & 1.10 \\
\hline & 0.92 & & 0.85 \\
\hline & $0.64 * *$ & & $0.35 * * *$ \\
\hline & $2.77^{* * *}$ & & 1.12 \\
\hline & 1.00 & & 1.00 \\
\hline & $0.57^{* *}$ & & $0.56^{* * *}$ \\
\hline & 0.34 & & 0.40 \\
\hline & 1.00 & & 1.00 \\
\hline & $0.49^{* * *}$ & & $0.55^{* * *}$ \\
\hline & 1.00 & & \\
\hline & $2.26 * * *$ & & $1.50^{* * *}$ \\
\hline & 1.00 & & \\
\hline & $2.27^{* * *}$ & & $1.40 * *$ \\
\hline & 1.00 & & $1.00 * *$ \\
\hline & & & 0.95 \\
\hline & & & 1.00 \\
\hline & & & 0.76 \\
\hline & & & 0.97 \\
\hline & & & 0.83 \\
\hline & & & 1.00 \\
\hline & & & 0.89 \\
\hline & & & $\begin{array}{l}1.511^{* *} \\
1.00\end{array}$ \\
\hline & & & 0.88 \\
\hline
\end{tabular}

Annexe. Tableau B. Facteurs associés à la connaissance, l'utilisation et les intentions d'utiliser la contraception moderne au Ghana (EDS 2008). Régression logistique par blocs (nested): $\beta_{0}+\beta_{1} x_{1}+\ldots+\beta_{n} x_{n}=\ln (P x / I-P x)$. Valeurs fournies : odd ratio (Exp du coefficient $\beta$ ). 


\begin{tabular}{|c|c|c|c|c|c|c|c|c|c|c|c|c|c|}
\hline \multirow{5}{*}{ 窝出离 } & \multicolumn{8}{|c|}{ GHANA - FEMMES } & \multicolumn{5}{|c|}{ GHANA - HOMMES } \\
\hline & \multirow{3}{*}{ Résidence } & \multicolumn{2}{|c|}{ CONNAISSANCE } & \multicolumn{3}{|c|}{ UTILISE AU MOINS UNE FOIS } & \multicolumn{2}{|c|}{ INTENTION } & \multicolumn{2}{|c|}{ CONNAISSANCE } & \multicolumn{3}{|c|}{ UTILISE AU MOINS UNE FOIS } \\
\hline & & 1.49 & 0.68 & $1.17 *$ & 0.95 & 0.97 & 0.81 & $0.72 * *$ & 2.43 & 1.57 & $1.54^{* * *}$ & 0.97 & 0.95 \\
\hline & & 1.00 & 1.00 & 1.00 & 1.00 & 1.00 & 1.00 & 1.00 & 1.00 & 1.00 & 1.00 & 1.00 & 1.00 \\
\hline & $\begin{array}{r}\text { capitale, grande vill } \\
\text { petite ville } \\
\text { campagnes }\end{array}$ & $0.27^{* * *}$ & 1.15 & $0.68 * * *$ & 1.08 & 1.07 & $1.32 * * *$ & $1.39 * *$ & $0.42 * * *$ & 1.20 & $0.41 * * *$ & 0.94 & 0.94 \\
\hline \multirow{29}{*}{ 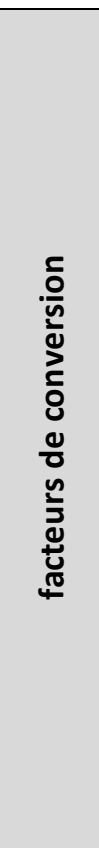 } & Ethnie & & 1.00 & & 1.00 & 1.00 & & 1.00 & & 1.00 & & 1.00 & 1.00 \\
\hline & Ga/Dangme & & $0.45 *$ & & $1.28 *$ & $1.28 *$ & & 0.88 & & $0.12 * * *$ & & $1.45 * *$ & $1.45 * *$ \\
\hline & Ewe & & 1.03 & & $1.31 * * *$ & $1.34 * * *$ & & $0.72 *$ & & $0.11 * * *$ & & $1.48^{* * *}$ & $1.51 * * *$ \\
\hline & Mole-Dagbani & & $0.59 *$ & & $1.25 * *$ & $1.34 * * *$ & & 0.91 & & $0.15 * * *$ & & 0.96 & 1.04 \\
\hline & Autre & & $0.29 * * *$ & & 0.86 & 0.90 & & 1.07 & & $0.12 * * *$ & & $0.82 *$ & 0.87 \\
\hline & Richesse ménage (quintile) & & $0.33^{* * *}$ & & $0.64 * * *$ & $0.64^{* * *}$ & & 1.08 & & 0.69 & & $0.59^{* * *}$ & $0.62 * * *$ \\
\hline & pauvre & & 0.77 & & 0.85 & 0.84 & & 0.95 & & 0.82 & & 0.90 & 0.91 \\
\hline & moyen & & 1.00 & & 1.00 & 1.00 & & 1.00 & & 1.00 & & 1.00 & 1.00 \\
\hline & riche & & 1.49 & & 1.14 & 1.16 & & 0.95 & & 1.55 & & $1.33 * *$ & $1.30 * *$ \\
\hline & plus riche & & $4.15 * *$ & & 1.20 & $1.25 *$ & & 1.24 & & 0.86 & & $1.97^{* * *}$ & $1.89 * * *$ \\
\hline & Groupe d'âge & & $0.37^{* * *}$ & & $0.63 * * *$ & $0.63^{* * *}$ & & 1.23 & & $0.34^{* * *}$ & & $0.48^{* * *}$ & $0.48 * * *$ \\
\hline & 20-29 & & 1.00 & & 1.00 & 1.00 & & 1.00 & & 1.00 & & 1.00 & 1.00 \\
\hline & 30-39 & & 1.40 & & 1.05 & 0.98 & & $0.63 * * *$ & & 1.55 & & 0.87 & 0.87 \\
\hline & $\begin{array}{r}40-49 \\
50-59\end{array}$ & & 1.01 & & $0.84 *$ & $0.70^{* * *}$ & & $0.13 * * *$ & & 0.70 & & $0.49^{* * *}$ & $0.49 * * *$ \\
\hline & marié(e)/cohabitant(e) & & 1.50 & & 1.16 & 1.18 & & 1.11 & & $2.17 *$ & & $0.36+90$ & 0.87 \\
\hline & célibataire/divorcé(e)/veuve-veuf & & 1.00 & & 1.00 & 1.00 & & 1.00 & & 1.00 & & 1.00 & 1.00 \\
\hline & Statut dans ménage chef(fe) & & $3.42 * *$ & & 0.94 & 0.97 & & 0.84 & & 1.83 & & 0.81 & 0.82 \\
\hline & époux/se & & 0.91 & & 0.96 & 0.98 & & 1.02 & & 0.35 & & 0.61 & 0.62 \\
\hline & autre & & 1.00 & & 1.00 & 1.00 & & 1.00 & & 1.00 & & 1.00 & 1.00 \\
\hline & Scolarisation & & $0.43^{* * *}$ & & $0.55^{* * *}$ & $0.54^{* * *}$ & & 1.01 & & $0.40^{* * *}$ & & $0.40^{* * *}$ & $0.41 * * *$ \\
\hline & primaire (qques années ou achevé) & & 1.00 & & 1.00 & 1.00 & & 1.00 & & 1.00 & & 1.00 & 1.00 \\
\hline & secondaire et plus & & $2.00 * *$ & & 1.14 & $1.19 * *$ & & 1.18 & & $3.13 * * *$ & & $1.32 * *$ & $1.29 * *$ \\
\hline & Activité prof & & 0.77 & & 0.90 & 0.93 & & 0.89 & & 0.93 & & 0.82 & 0.80 \\
\hline & non-manuelle & & 1.13 & & $1.18 *$ & $1.24 * *$ & & 1.00 & & 1.44 & & $1.46^{* * *}$ & $1.43 * * *$ \\
\hline & agriculture & & 1.00 & & 1.00 & 1.00 & & 1.00 & & 1.00 & & 1.00 & 1.00 \\
\hline & manuelle & & 1.36 & & 1.18 & 1.24 * & & 1.06 & & 0.99 & & $1.29 * *$ & $1.27 * *$ \\
\hline & $\mathrm{Nb}$ enfants vivants & & & & & 1.00 & & 1.00 & & & & 1.00 & 1.00 \\
\hline & $4-6$ & & & & & $1.62 * * *$ & & $1.54 * * *$ & & & & 1.07 & 1.08 \\
\hline & $7+$ & & & & & $2.37^{* * *}$ & & 1.37 & & & & 0.88 & 0.97 \\
\hline \multirow{7}{*}{ 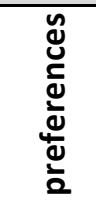 } & Souhait enfant suppl & & & & & $0.83 * *$ & & 0.85 & & & & & 1.21 \\
\hline & dans les 2 ans & & & & & $0.69 * * *$ & & $0.77 *$ & & & & & 0.96 \\
\hline & au-delà de 2 ans & & & & & 1.00 & & 1.00 & & & & & 1.00 \\
\hline & souhaite, NSP quand & & & & & 1.06 & & $0.64 * *$ & & & & & 1.69 \\
\hline & Nb idéal d'enfants & & & & & 0.99 & & 1.20 & & & & & $1.20 *$ \\
\hline & $4-6$ & & & & & 1.00 & & 1.00 & & & & & 1.00 \\
\hline & $7+$ & & & & & $0.64 * * *$ & & $0.70 *$ & & & & & $0.62 * * *$ \\
\hline
\end{tabular}


Annexe. Tableau C.

Intervalles proto- et inter-génésiques et culture contraceptive: populations de référence

\begin{tabular}{|c|c|c|c|}
\hline & \multirow[t]{2}{*}{ Population de référence } & \multicolumn{2}{|c|}{ Effectifs de femmes } \\
\hline & & MALI & GHANA \\
\hline $\begin{array}{l}\text { Intervalle proto- } \\
\text { génésique (mariage - 1è } \\
\text { naissance) }\end{array}$ & $A$ au moins un enfant & $10^{\prime} 457$ & 2,873 \\
\hline Contraception : & & & \\
\hline -Déjà utilisée & +connaissance et déjà utilisé & 1929 & 1194 \\
\hline -Jamais utilisée, mais & + connaissance, jamais utilisé, mais & 1880 & 488 \\
\hline intention & intention & & \\
\hline -Jamais utilisée, pas & +connaissance, jamais utilisé, pas & 6648 & 991 \\
\hline l'intention & l'intention & & \\
\hline $\begin{array}{l}\text { 1er intervalle inter- } \\
\text { génésique } \\
\text { (1è-2è naissance) }\end{array}$ & A au moins deux enfants & 9372 & 2648 \\
\hline Contraception : & & & \\
\hline -Déjà utilisée & + connaissance et déjà utilisé & 1918 & 1237 \\
\hline -Jamais utilisée, mais & + connaissance, jamais utilisé, mais & 1654 & 439 \\
\hline intention & intention & & \\
\hline -Jamais utilisée, pas & +connaissance, jamais utilisé, pas & 6160 & 972 \\
\hline l'intention & l'intention & & \\
\hline $\begin{array}{l}\text { 2è intervalle inter- } \\
\text { génésique } \\
\text { (2è-3è naissance) }\end{array}$ & A au moins trois enfants & 8021 & - \\
\hline Contraception & & & - \\
\hline -Déjà utilisée & + connaissance et déjà utilisé & 1588 & - \\
\hline -Jamais utilisée, mais & +connaissance, jamais utilisé, mais & 1329 & - \\
\hline intention & intention & & \\
\hline -Jamais utilisée, pas & +connaissance, jamais utilisé, pas & 5104 & - \\
\hline l'intention & l'intention & & \\
\hline
\end{tabular}


African Population Studies Vol 28, No.2 June 2014

Annexe. Tableau D. Facteurs associés à l'espacement des naissances

(Probabilité d'être parmi les femmes du tercile le plus long)

Régression logistique par blocs (nested): $\beta_{0}+\beta_{1} x_{1}+\ldots+\beta_{n} x_{n}=\ln (P x / I-P x)$.

Valeurs fournies : odd ratio (Exp du coefficient $\beta$ ).

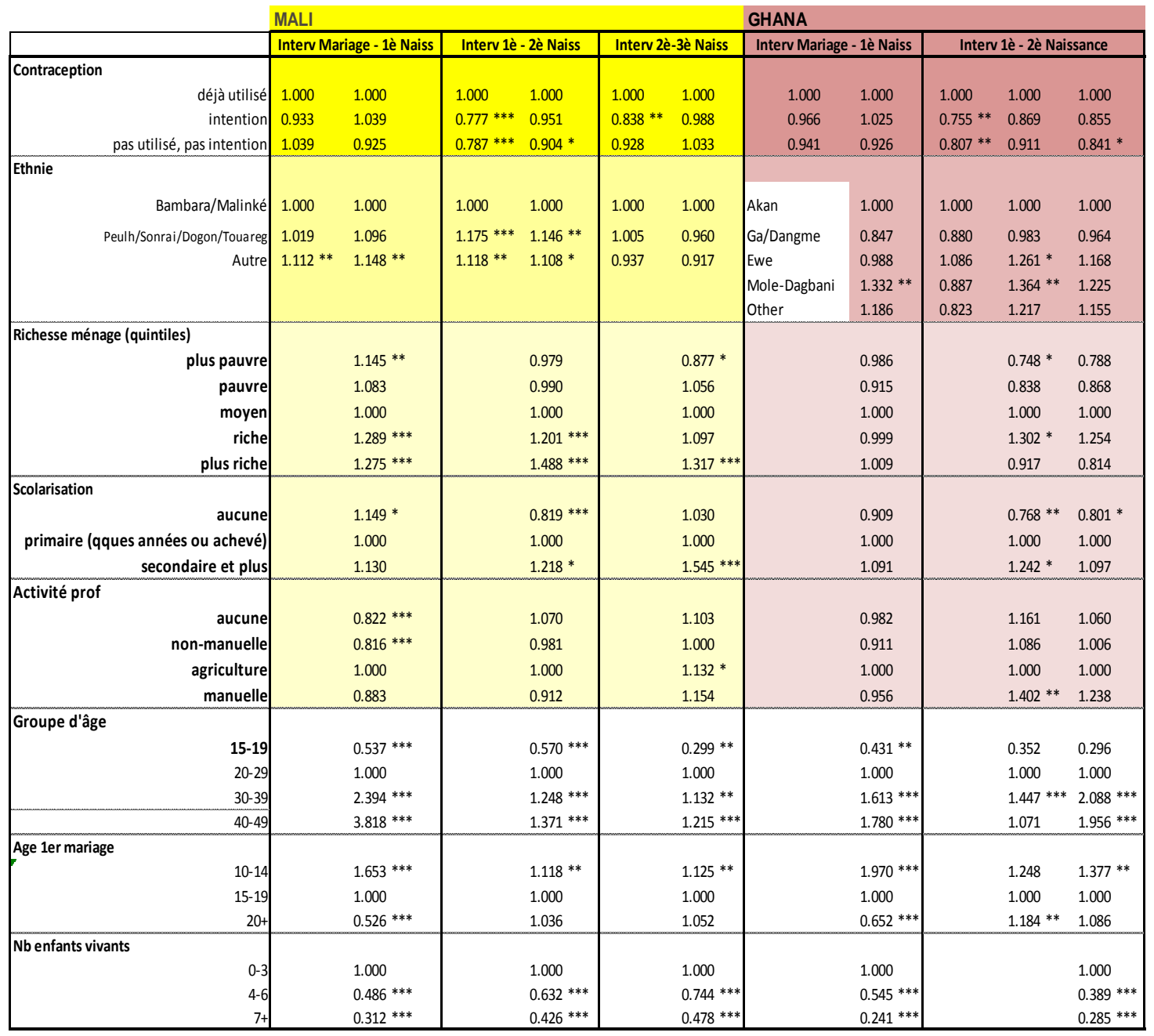


${ }^{1}$ http://www.familyplanning2020.org/index.php

${ }^{2}$ Voir le site web de MEASURE DHS, http://measuredhs.com/Topics/Unmet-Need.cfm.

${ }^{3}$ Theory of Planned Behaviour (Ajzen I., 1988), appliquée aux intentions de fécondité notamment par Billari et al (2009).

${ }^{4}$ Indice EDS calculé sur la base de la présence dans le ménage d'électricité, eau courante, type de toilettes, revêtement du sol, cuisine et de biens (media, transport), Rutstein and Johnson (2004).

${ }^{5}$ Implicite est donc le postulat que, globalement, les grossesses et naissances prémaritales précoces participent aussi de cette culture familialiste.

${ }^{6}$ Nous n'abordons pas ici les raisons du non-recours à la contraception, mais les facteurs qui accroissent ou diminuent l'accès à la connaissance et la capacité de pratique.

${ }^{7}$ L'enquête du Ghana ne comporte pas d'information sur les infrastructures disponibles.

${ }^{8}$ Question non posée aux hommes.

ix En outre, on constate que la prise en compte des comportements contraceptifs ne modifie pas l'effet des caractéristiques personnelles : la régression sans la variable contraception (non reproduite dans le tableau) donne des coefficients similaires pour les facteurs de conversion.

x L'appartenance ethnique ne jouant pas de rôle pour le second intervalle.

xi La taille de la famille n'est donc pas considérée comme un facteur causal de la durée des intervalles ce qui constituerait une incohérence chronologique. 